\title{
Searching for the Achilles' heel of cancer
}

\author{
Myeloproliferative neoplasms in the era of precision medicine
}

\author{
Klaus Geissler
}

Received: 21 November 2016 / Accepted: 22 November 2016 / Published online: 30 November 2016 (C) Springer-Verlag Wien 2016

Targeted treatment of cancer has been a vision for hemato-oncologists for many years. Among the myeloproliferative neoplasms (MPNs) chronic myeloid leukemia (CML) was the first disease in which this dream has come true and in the other MPN entities we try to mimic this success.

CML was the first malignancy in which imatinib, a tyrosine kinase inhibitor (TKI) which inhibits the pathophysiologically crucial BCR-ABL protein, was able to profoundly inhibit the growth of the malignant clone and to greatly improve the prognosis of patients. Today imatinib and other TKIs such as nilotinib, dasatinib, bosutinib and ponatinib are well established in the treatment of CML, the survival of patients is approaching the survival of normal individuals and continuing molecular remissions can be observed in some patients even after discontinuation of treatment. In the issue 4/2016 of memo the pathophysiology and current treatment of CML is covered by articles of Gerald Webersinke [1] and Stefan Schmidt [2], respectively.

Among the BCR-ABL-negative NPMs, in which the JAK2 V617F mutation is the most common genetic abnormality, the JAK1/2 inhibitor ruxolitinib has been demonstrated to improve constitutional symptoms and splenomegaly in patients with intermediate2 or high-risk myelofibrosis and studies are beginning to show a survival benefit over patients who received placebo or best available therapy. Recently it was shown that patients with hydroxyurea-refractory or hydroxyurea-intolerant polycythemia vera also seem to benefit from ruxolitinib since the number of

\section{K. Geissler, MD ( $\bowtie)$}

5th Department of Internal

Medicine-Oncology/Hematology, Hospital Hietzing,

Wolkersbergenstraße 1, 1130 Vienna, Austria

klaus.geissler@wienkav.at phlebotomies and disease-related symptoms can be greatly reduced with this drug.

In contrast to the other MPNs treatment options in the myeloproliferative-chronic myelomonocytic leukemia (MP-CMML) are still unsatisfactory. The article by Sonja Burgstaller [3] clearly indicates that this disease deserves more attention, since some patients have an extremely poor prognosis and trials investigating new agents are still limited. For the design of such trials agents could be selected on the basis of new insights into the molecular pathophysiology which is discussed in the article by Klaus Geissler [4]. In particular, the RAS signaling pathway has recently been shown to play a major pathophysiologic role in MP-CMML and this knowledge holds great promise for the use of RAS pathway inhibitors in this entity.

The remarkable success that has been achieved in MPNs by TKIs is not only beneficial for patients suffering from these hematologic diseases but also provides the basis for hope in many other cancer patients, since it can be considered as a proof of the concept that at least some cancers can be successfully treated if the molecular pathophysiology of the disease can be elucidated which may result in the detection of the Achilles' heel of the malignancy.

Conflict of interest K. Geissler declares that he receives honoraria for presentations and advisory boards from Novartis, Bristol-Myers Squibb, Pfizer, and Incyte.

\section{References}

1. Webersinke G. Molecular pathogenesis of chronic myeloid leukemia. Memo. 2016; doi:10.1007/s12254-016-0294-0.

2. SchmidtS. Treatment of chronic myeloid leukemia. Memo. 2016; doi:10.1007/s12254-016-0294-0. 


\section{editorial}

3. Burgstaller S. Treatment of chronic myelomonocytic leukemia. Memo. 2016; doi:10.1007/s12254-016-0291-3.

4. Geissler K. Molecular pathogenesis of chronic myelomonocytic leukemia. Memo. 2016; doi:10.1007/s12254-0160295-z.

- For latest news from international oncology congresses see: http://www.springermedizin.at/ memo-inoncology 